\title{
AUTOMATIZAÇÃO DE UM BANHEIRO PÚBLICO PARA REDUÇÃO DA CONTAMINAÇÃO POR DOENÇAS INFECCIOSAS
}

\section{TITLE: AUTOMATIZATION OF A PUBLIC TOILET FOR THE REDUCTION OF INFECCIOUS DISEASES CONTAMINATION REDUCTION}

\author{
Gabriel Liobino Sampaio ${ }^{\mathrm{I}}$ \\ Guilherme Matheus Rafael Parcerão ${ }^{I I}$ \\ Isabella Romão Chaves ${ }^{\mathrm{III}}$ \\ Fernando Luis de Almeida ${ }^{\text {IV }}$
}

\begin{abstract}
RESUMO
Com a pandemia da Coronavirus disease 2019 (Covid-19), que assola o mundo, o contato direto em ambientes propícios à contaminação deve ser evitado; nos quais, o ambiente sanitário se mostra como um local com mais chances de ocorrer uma contaminação, sem que o usuário a perceba. Assim, o principal objetivo do presente trabalho de graduação é automatizar um banheiro de forma a evitar a contaminação e favorecer o saneamento e a acessibilidade, promovendo a saúde pública. Em relação aos métodos, foi adotada uma pesquisa exploratória com uma abordagem hipotético-dedutiva, e um procedimento experimental que represente um banheiro de uso público. A automatização foi realizada de acordo com os métodos já existentes para automatizar os componentes de um banheiro, sendo estes a possibilidade de erguer a tampa do sanitário e acionar uma torneira, ambos de maneira automatizada e sem a necessidade de contato humano. Em seguida, revisou-se as propostas já existentes dentro do tema "banheiro automatizado", como a automatização de elementos como portas, dispensers etc., com objetivo de encontrar viabilidade em sua aplicação neste projeto reduzindo seus gastos. A segunda etapa consiste na elaboração do design eletrônico e na programação dos processos. Foi possível realizar a automatização da torneira e do vaso sanitário, assim sendo, uma automatização parcial do banheiro por meio deste trabalho. Espera-se que com os resultados obtidos, a automatização do banheiro público possa contribuir com o saneamento e a melhora da saúde.
\end{abstract}

Palavras-chave: Saneamento. Automatização. Acessibilidade. Saúde. Banheiro.

\begin{abstract}
With the pandemic of Coronavirus disease 2019 plaguing the world, direct physical contact in environments conductive to contamination must be avoided; in which the sanitary environment shows itself as a local with greater chances to occur contamination without the user's perception. Along this, this present graduation project main objective is to automatize a toilet

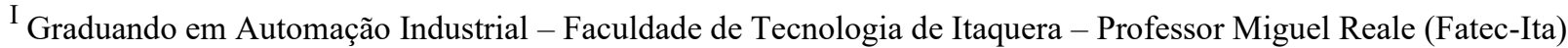
- São Paulo - Brasil. E-mail: gabriel.sampaio2@fatec.sp.gov.br

II Tecnólogo em Automação Industrial - Faculdade de Tecnologia de Itaquera - Professor Miguel Reale (Fatec-Ita) - São Paulo - Brasil. E-mail: guilherme.parcerao@fatec.sp.gov.br

III Tecnóloga em Automação Industrial - Faculdade de Tecnologia de Itaquera - Professor Miguel Reale (Fatec-Ita) - São Paulo - Brasil. E-mail: isa_crc@hotmail.com

IV Prof. Pós-Dr. em Engenharia Elétrica na Faculdade de Tecnologia de Itaquera - Professor Miguel Reale (FatecIta) - São Paulo - Brasil. E-mail: fernando.almeida14@fatec.sp.gov.br
} 


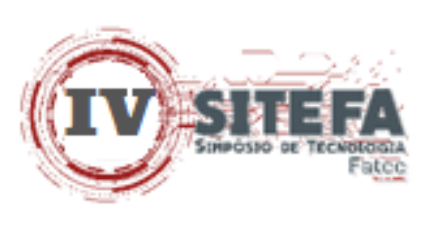

to avoid contamination and improve sanitation and accessibility, promoting public health. In relation to the methods, exploratory research was used with a hypothetical-deductive approach, and an experimental procedure to represent a public toilet. The automation used following existing methods to automatize the toilet components, being those the possibility of lift the sanitary cover and trigger a tap, both with automation without necessity of physical human contact. Following, the existing purposes for the "automatized bathroom" theme were revised, like doors, dispensers etc., with objective for find the project's viability in this project to reduce their costs. The second step consists on the development of the electronic design and software programming. It was possible to make the tap and sanitary partial automation through this project. With the obtained results, the expectation is that the public toilet automation can contribute with the sanitation and public health.

Keywords: Sanitation. Automation. Accessibility. Health. Toilet.

Data de submissão do artigo: 31/05/2021.

Data de aprovação do artigo: 06/07/2021.

DOI: $10.33635 /$ sitefa.v4i1.159

\section{INTRODUÇÃO}

Com a chegada da Internet das Coisas, a área da microeletrônica obteve inúmeros avanços. Com isso, fora permitido que os conceitos de automatização ganhassem novas aplicações na área de estudo de saneamento, sendo responsável por diversas contribuições na prevenção de doenças, em especial em locais que atuam como vetores de doenças contagiosas, como o banheiro público.

Com isso, entendemos que o banheiro público pode se tornar um ambiente altamente propício à contaminação, uma vez que não é higienizado após o uso de cada indivíduo, tornando o ambiente volúvel aos vírus.

Outro ponto necessário a se levar em consideração é o estudo de Silva et al. (2013), em que se constatou que ovos do verme Enterobius vermicularis estavam presentes em sua maior parte, nas maçanetas internas e externas das portas dos sanitários públicos de cinco Unidades Básicas de Saúde, demonstrando grande risco de contaminação.

No ano de 2020, surgiu um novo vírus com altíssima capacidade de contaminação e taxa de fatalidade, o que gerou uma crise econômica e sanitária generalizada e causou a morte de 2 milhões de pessoas, segundo dados da Center for Systems Science and Engineering at Johns Hopkins University (2021). Com isso, vivenciamos um momento ímpar em nossa história, não apenas em nosso país, mas em todo o mundo.

A contaminação dessas doenças ocorre por meio do contato direto, seja com os fluidos, tato ou o contato com espirros e tosses, que também são meios viáveis para o contágio; com isso, a higienização das mãos e de ambientes de uso coletivo torna-se extremamente importante. Em razão disso é preciso evitar o contato direto das pessoas com os agentes transmissores de doenças causadas por bactérias e vírus.

Assim, levanta-se a seguinte questão investigativa: é possível reduzir o contágio dentro um banheiro público a partir da automatização?

A resposta pode advir deste trabalho que propõe a automatização de um banheiro público com base em três pilares: saúde, acessibilidade e sustentabilidade. Também faz parte 


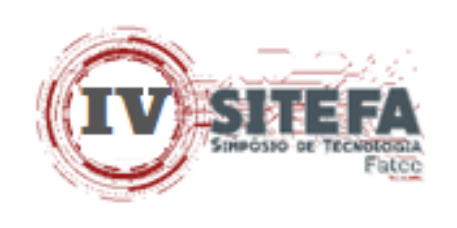

da proposta a redução no número de contaminações por meio de doenças virais que se transmite pelo toque.

Por fim, além da redução de contágio, tais medidas também podem auxiliar na economia de água, pois as descargas serão dadas apenas quando for necessário e na quantidade correta por meio de uma redundância, e energia elétrica, fazendo com que as luzes do local se acendam apenas com a presença de uma ou mais pessoas.

\section{AUTOMAÇÃO PREDIAL}

Rosário (2009) define a automatização como todo processo que realiza tarefas e atividades de forma autônoma ou que oferece auxílio em tarefas manuais. Com o surgimento da Indústria 4.0, as tecnologias industriais começaram a se integrar cada vez mais, até que o termo também passou a ser utilizado para designar a integração entre sistemas industriais. $\mathrm{O}$ trabalho de Lins e Moura (2009) aponta que a automação predial está dividida em dois tipos de arquiteturas, arquitetura baseada em automação (ABA), conhecida como automação estática e a arquitetura baseada em comportamento (ABC), também conhecida como automação inteligente. A automação predial que utiliza a arquitetura $\mathrm{ABA}$, trata a automação de residências a partir de dispositivos remotos de modo que todos esses possam ser ajustados e configurados pelos seus usuários, de modo que, os habitantes da residência não tenham que se adaptar ao sistema nem precisar configurá-lo, fazendo com que o mesmo funcione de acordo com suas necessidades. A automação predial baseada na arquitetura $\mathrm{ABC}$, chamada de "automação inteligente" (entende-se por automação inteligente o processo de automação que incorpore algum mecanismo automático de tomada de decisão baseada em técnicas de inteligência artificial) utiliza algoritmos como o ID3.

\subsection{ESP32}

Para as referências nos trabalhos de IoT (do inglês Internet of Things - Internet das Coisas) com ESP32, pode-se citar o trabalho de Aghenta e Iqbal (2019), que consiste no desenvolvimento de um sistema de supervisão de baixo custo com a utilização da arquitetura SCADA e ESP32, de Rai e Rehman (2019), que consiste na montagem de um sistema de supervisão com módulos Adafruit.

\subsection{Estado da arte}

Dentre os outros trabalhos realizados sobre esse mesmo tema, pode-se citar os banheiros inteligentes de Dubai. Segundo Serkal (2019), esses banheiros vêm equipados com touchscreens com instruções em árabe e inglês e um sistema programado para dedetizar o banheiro com água e desinfetantes durante um minuto após cada uso.

Também é possível citar o o trabalho de Gambhir que foi noticiado por Armitage (2020), que trata de um sistema capaz de detectar doenças presentes nos dejetos, como cânceres colorretais e urológicos, por meio de diversas tecnologias como sensoriamento de movimento e análises físicas e moleculares, todas conectadas à um sistema de armazenamento em nuvem capaz de armazenar todos os dados de maneira segura. Os dejetos foram capturados em vídeo e analisados por um algoritmo que possui como função detectar alterações em sua dinâmica. 


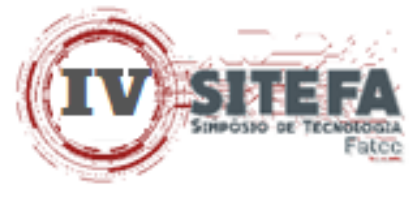

\section{MATERIAIS E MÉTODOS}

Para a realização do projeto, é necessário o uso de um microcontrolador para processar os dados advindos dos sensores e acionar os atuadores conforme a lógica desenvolvida. Para tal, foi escolhido o ESP32, que é um microcontrolador desenvolvido pela empresa chinesa Espressif. De acordo com Maier (2017), ele possui arquitetura 32 bits e processador dualcore com clock máximo de $240 \mathrm{MHz}$, além de $520 \mathrm{kB}$ de RAM, $448 \mathrm{kB}$ de ROM, memória flash de $4 \mathrm{MB}$ e módulo $\mathrm{Wi}$-Fi. Sua programação é realizada pela biblioteca Wire por meio da IDE Arduino, sendo assim de fácil programação e configuração, inclusive para pessoas com pouco conhecimento em programação. A biblioteca Wire consiste em uma linguagem de programação desenvolvida para o Arduino e outras plataformas baseadas no mesmo, como a plataforma $\mathrm{ESP} /$ NodeMCU, e é formada por meio de uma mistura de elementos das principais linguagens de programação do mercado.

Para realizar o desenvolvimento do protótipo, foram utilizados 2 emissores infravermelhos, 2 receptores infravermelhos, 2 válvulas solenoides $1 / 2$ X 1/2, 30 resistores cerâmicos $1 / 4 \mathrm{~W}, 1$ sensor reflexivo infravermelho, 1 sensor de peso, 1 motor de passo, 1 sensor magnético com fio de sobrepor, 1 módulo NodeMCU ESP32, 2 sensores de temperatura DS18B20, 1 módulo amplificador HX711, 1 módulo relé optoacoplado, 1 driver A4498 e 4 módulos LDR.

O estudo utilizou de uma pesquisa do tipo exploratória. Segundo Gil (2002), a pesquisa exploratória possui a finalidade de proporcionar maior familiaridade com o problema, geralmente envolvendo levantamento bibliográfico. Este trabalho envolveu uma pesquisa bibliográfica para levantar o que já foi feito na temática do trabalho e as lacunas existentes, além de aumentar a familiaridade com o tema interdisciplinar da pesquisa.

A pesquisa usou de uma abordagem hipotético-dedutiva. De acordo com Marconi e Lakatos (2003), a abordagem hipotético-dedutiva deve conter a colocação do problema e estabelecimento dos fatos, que é o risco de contaminação, a construção de um modelo teórico e das hipóteses, a dedução das consequências, o teste das hipóteses e as considerações finais juntamente com os resultados obtidos.

O estudo foi abordado como um procedimento experimental atribuído pela familiaridade na redução da transmissão de doenças em um ambiente sanitário, buscando um aprimoramento na redução de custo no desenvolvimento do protótipo, onde os resultados apresentados por este são atribuídos por procedimentos empíricos e experimentais. A pesquisa experimental caracteriza-se por manipular diretamente as variáveis relacionadas com o objeto de estudo. Portanto, a pesquisa experimental pretende dizer de que modo ou por que causas o fenômeno é produzido (RODRIGUES JUNIOR, 2021). Neste caso, o protótipo e as observações determinadas pelo método científico serão realizados de acordo com dados coletados dos sensores e testes empíricos realizados no protótipo físico.

A principal finalidade do procedimento experimental é testar hipóteses para comprovar a convicção do pesquisador envolvendo grupos de controle, seção aleatória e manipulação de variáveis, buscando-se técnicas de coleta de amostragem realizadas durante o experimento, assim manipulando diretamente as variáveis relacionadas ao objeto de estudo. Aplicado à pesquisa, o conceito de pesquisa experimental será utilizado na coleta de dados advindos dos sensores utilizados para desenvolver um sistema eficiente e funcional. 


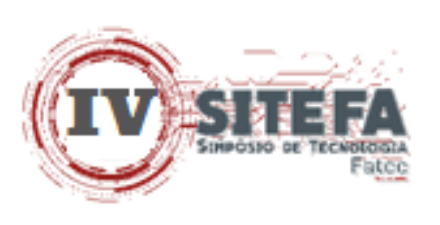

\section{DESENVOLVIMENTO}

Primeiramente, foi realizada uma pesquisa técnico-científica para encontrar outros trabalhos que possuíam um tema semelhante. Por meio desta pesquisa, foi verificado que a maior parte dos projetos de mesma temática possuem custo muito elevado e inacessível para a população média. Em seguida, foi realizada uma pesquisa de mercado para levantar o material necessário para a construção do protótipo e os gastos necessários, bem como os softwares necessários para a realização do mesmo, como o IDE Arduino.

Posteriormente, foram elaborados os designs eletrônicos necessários para o funcionamento do protótipo e a programação necessária. Para tal, cada sensor utilizado foi testado individualmente e foi notado que nenhum necessitava de algum ajuste ou tratamento adicional, pois a interface oferecida pelos módulos e pelos pinos de I/O do ESP32, bem como seus circuitos de pull-in e pullout, modularam o sinal de maneira suficiente para sua utilização pretendida. As ligações foram realizadas de acordo com o diagrama de blocos apresentado na Figura 1.

Figura 1 - Diagrama de blocos das ligações elétricas

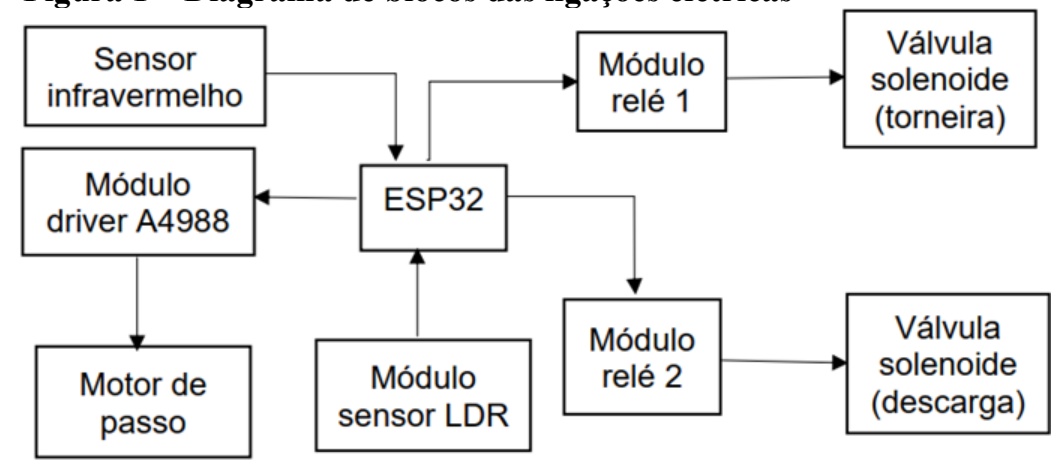

Fonte: elaborado pelos autores (2020)

Por fim, deu-se início à etapa de construção do protótipo.

Primeiramente, foi realizada a solda de fios macho-macho utilizando ferro de solda e estanho nas saídas de $12 \mathrm{~V}, 5 \mathrm{~V}$ e terminal terra de uma fonte de $500 \mathrm{~W}$.

Em seguida foram soldados fios nos terminais das válvulas solenoide, enquanto o ESP32 foi anexado à placa e alimentado com $5 \mathrm{~V}$. Então, o módulo A4988 para controle do motor de passo foi anexado e alimentado com $12 \mathrm{~V}$ e foi colocado um capacitor de $220 \mu \mathrm{F}, 35 \mathrm{~V}$ em paralelo com a alimentação conforme a ligação apresentada na Figura 2.

Figura 2 - Ligação elétrica do motor de passo

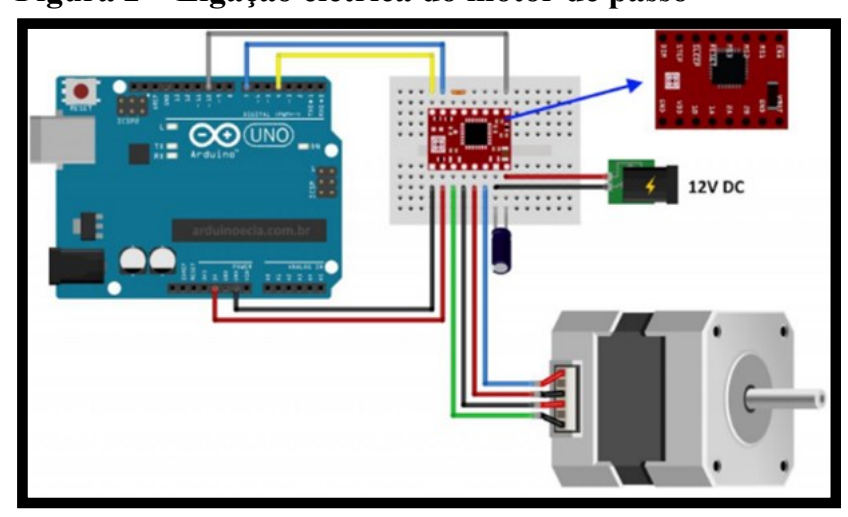

Fonte: Arduino e Cia (2015)

Simpósio de Tecnologia (Sitefa) - Fatec Sertãozinho - SP, v. 4, n. 1, p. 176-186, 2021. ISSN 2675-7540 


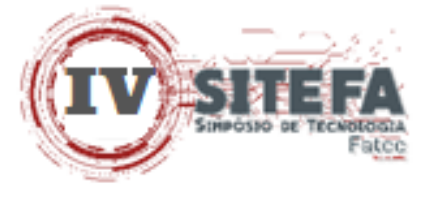

Os terminais do motor de passo e as saídas do ESP32 foram devidamente ligadas ao módulo para o controle do motor, conforme a Figura 3.

Figura 3 - Ligação do motor de passo com o módulo

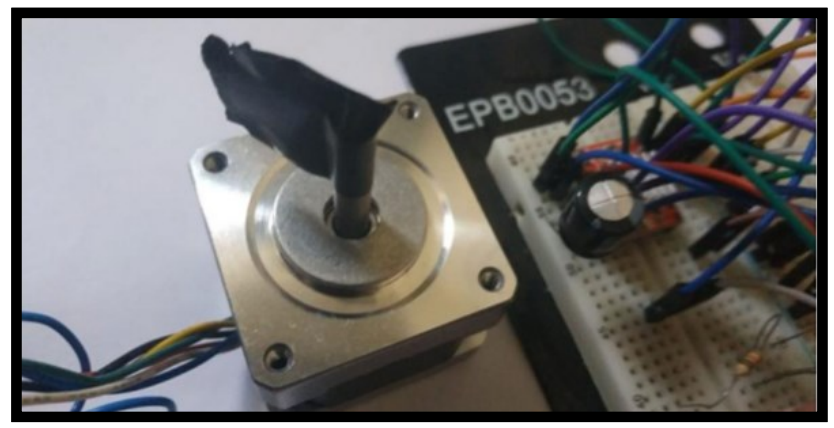

Fonte: elaborado pelos autores (2020)

Com isso, os sensores LDR e infravermelho foram conectados às entradas do ESP32. Os relés foram alimentados com $12 \mathrm{~V}$ para fornecer às válvulas solenoide a potência necessária, conforme Figura 4.

Figura 4 - Ligação do módulo ESP32

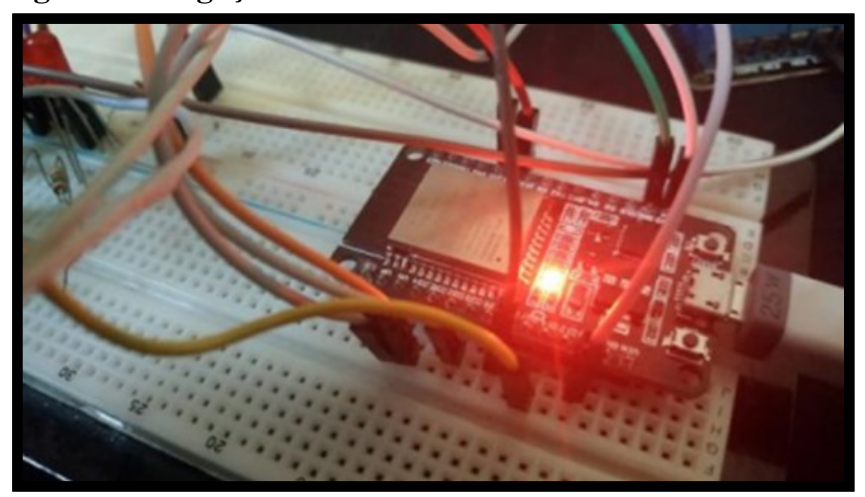

Fonte: elaborado pelos autores (2020)

As válvulas solenoide foram conectadas aos relés e ao neutro, conforme a Figura 5.

Figura 5 - Ligação da válvula solenoide

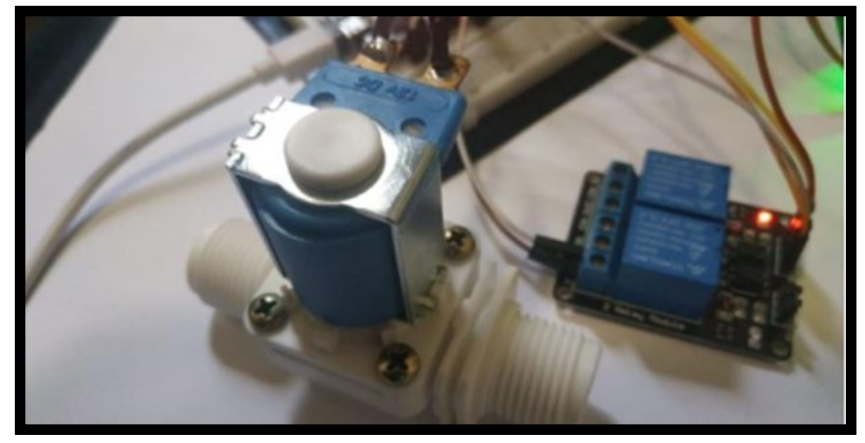

Fonte: elaborado pelos autores (2020) 


\section{(iv)}

A programação foi realizada através da biblioteca Wire na plataforma Arduino.

\section{RESULTADOS E DISCUSSÃO}

Foi possível realizar uma automatização parcial do ambiente sanitário por meio deste trabalho, com a automatização da torneira e do vaso sanitário tendo sido realizada. Entretanto, devido às limitações no orçamento algumas funções do vaso sanitário não foram aplicadas utilizando os sensores corretos. Não foram realizadas automatizações nas demais áreas devido a restrições no orçamento. Também não foi possível realizar a automatização de um meio de limpeza do ambiente. Todas essas áreas podem ser estudadas e trabalhadas em um projeto futuro.

$\mathrm{Na}$ Tabela 1, estão apresentados os dados extraídos dos seguintes sensores: magnético, infravermelho e termistor na grandeza de medida do protocolo serial como resultados quantitativos.

Tabela 1 - Medição dos sensores

\begin{tabular}{l|l|l|l|l|l|l|l|l|l}
\hline Sensores & \multicolumn{2}{l}{ Saída serial } \\
\hline Magnético & 3799 & 3791 & 3791 & 3799 & 3797 & 3799 & 3759 & 3887 & 3815 \\
Infravermelho & 3734 & 3731 & 3731 & 3735 & 3743 & 3733 & 3743 & 3735 & 3719 \\
Termistor & 2133 & 2081 & 2093 & 2080 & 2077 & 2087 & 2091 & 2086 & 2085 \\
\hline
\end{tabular}

Fonte: elaborado pelos autores (2020)

Aplicando a teoria de erros, para obter os valores reais, primeiro foi calculado a média de cada uma das medidas seguindo as regras de arredondamento, onde valores maiores que 5 são arredondados para cima, pois os valores devem ser do tipo inteiro, seguindo a Equação (1):

$$
m=\frac{1}{n} \sum x_{i}
$$

Onde:

$\mathrm{m}$ - média.

$\mathrm{n}$ - número de amostras.

$\mathrm{xi}$ - valor da amostra.

Em seguida, foi realizado o cálculo do desvio padrão de cada amostra para a obtenção da incerteza dos resultados de acordo com a Equação (2);

$$
s=\sqrt{\frac{1}{n-1} \sum\left(x_{i}-m\right)^{2}}
$$

Onde:

s - desvio padrão da amostra.

Posteriormente, foram usados os valores do desvio padrão das amostras para o cálculo do desvio padrão das amostras em relação à média pela Equação (3): 


$$
\begin{aligned}
& \text { (IU) SIIEFA } \\
& s_{m}=\sqrt{\frac{1}{\sqrt{(n-1)} \sum\left(x_{i}-m\right)^{2}}}=\frac{s}{\sqrt{n}}
\end{aligned}
$$

Onde:

sm - desvio padrão da média.

Finalmente, o valor real das medidas foi demonstrado de acordo com a Equação (4):

$$
x=m_{i} \pm s_{m_{i}}
$$

Onde:

$\mathrm{x}$ - valor real da medida.

mi - média da medida.

smi - desvio padrão da média da medida.

Os resultados obtidos após a aplicação dos cálculos da Teoria de Erros, bem como o valor real aproximado, podem ser conferidos na Tabela 2:

Tabela 2 - Medição dos sensores

\begin{tabular}{c|l|l|l|l}
\hline Sensores & $\begin{array}{l}\text { Média } \\
\text { (serial) }\end{array}$ & $\begin{array}{l}\text { Desvio padrão } \\
\text { (serial) }\end{array}$ & $\begin{array}{l}\text { Desvio padrão da } \\
\text { média (serial) }\end{array}$ & $\begin{array}{l}\text { Valor real } \\
\text { (serial) }\end{array}$ \\
\hline Magnético & 3799 & 3791 & 3791 & $3804 \pm 11$ \\
Infravermelho & 3734 & 3731 & 3731 & $3734 \pm 2$ \\
Termistor & 2133 & 2081 & 2093 & $2090 \pm 6$ \\
\hline
\end{tabular}

Fonte: elaborado pelos autores (2020)

Em relação ao sensor de temperatura, foram realizados testes com água em temperatura natural e água com temperatura aquecida para simular a presença de dejetos na água. As medições obtidas se encontram na Tabela 3.

Tabela 3 - Medidas obtidas pelo sensor de temperatura

\begin{tabular}{c|l|l|l|l|l|l|l|l}
\hline Sensores & \multicolumn{2}{|l|}{${\text { Temperatura }\left({ }^{\circ} \mathbf{C}\right)}^{(14}$} & 14 & 14 & 14 & 14 & 14 & 14 \\
\hline Água natural & 14 & 14 & 14 & \\
Água aquecida & 25,93 & 25,74 & 26,64 & 25,77 & 25,82 & 25,97 & 25,88 & 25,89 \\
\hline
\end{tabular}

Fonte: elaborado pelos autores $(\mathbf{2 0 2 0})$

Aplicando a teoria de erros, foram obtidos os resultados demonstrados na Tabela 4.

Tabela 4 - Valores reais da temperatura da água de acordo com a teoria de erros

\begin{tabular}{c|l|l|l|l}
\hline Sensores & $\begin{array}{l}\text { Média } \\
\left({ }^{\circ} \mathbf{C}\right)\end{array}$ & $\begin{array}{l}\text { Desvio padrão } \\
\left({ }^{\circ} \mathbf{C}\right)\end{array}$ & $\begin{array}{l}\text { Desvio padrão da } \\
\text { média }\left({ }^{\circ} \mathbf{C}\right)\end{array}$ & Valor real $\left({ }^{\mathbf{0}} \mathbf{C}\right)$ \\
\hline Água natural & 14 & 0 & 0 & 14 \\
Água aquecida & 25,955 & 0,288 & 0,102 & $25,955 \pm 0,102$ \\
\hline
\end{tabular}

Fonte: elaborado pelos autores (2020) 


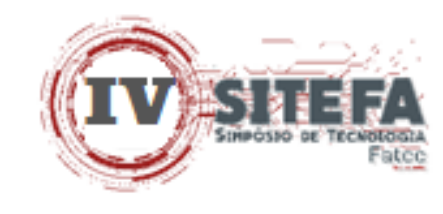

O protótipo final obtido para o kit para automatizar um vaso sanitário pode ser visto na Figura 6.

Figura 6 - Protótipo final do kit para automatizar um vaso sanitário

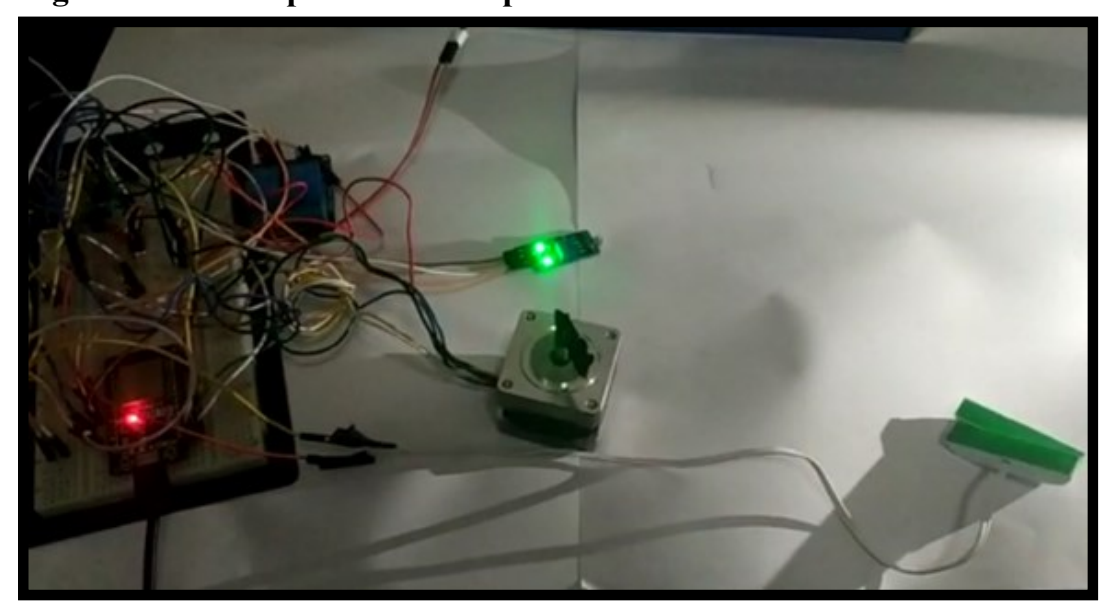

Fonte: elaborado pelos autores (2020)

O protótipo final para a torneira automatizada pode ser visto na Figura 7.

Figura 7 - Protótipo final da torneira automatizada
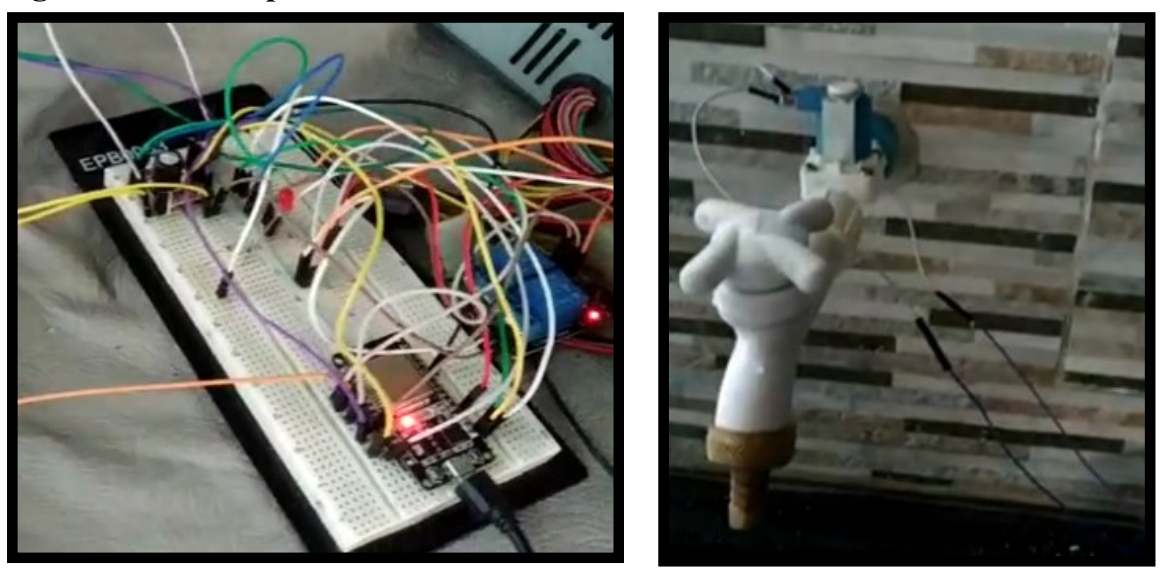

Fonte: elaborado pelos autores (2020)

\subsection{Escolha dos sensores}

Os sensores foram utilizados de acordo com as necessidades que surgiram na elaboração do protótipo e de acordo com os preços. Os sensores de Infravermelho e LDR foram utilizados para detectar presença, sendo possível aplicar ambos, mas sendo preferível o uso do sensor infravermelho. O sensor de temperatura W1209 foi utilizado para medição da temperatura da água no fundo do vaso sanitário para detectar se houve alteração na água que indique a urina na água, este sensor foi utilizado no lugar do sensor de turbidez que é usado para medir a turbidez da água, o que indica a presença de urina ou fezes na água, porém devido ao seu custo não pode ser implantado. 


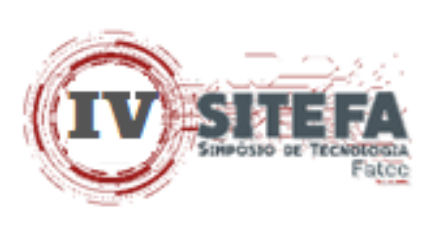

\subsection{Lógica de programação}

Um problema encontrado durante a programação foi a leitura incorreta de um dos sensores LDR gerando falsas leituras de presença do usuário, a substituição do sensor digital por um analógico permitiu impedir que este erro ocorresse por problemas no sensor.

Houve um erro na lógica utilizada para definir o tipo de descarga que seria utilizada pelo vaso sanitário, onde se levava em conta o usuário sentar-se no assento para definir se deveria ser realizada a descarga de 6 litros ou de 3 litros, essa lógica, porém, não pode ser aplicada à usuários de ambos os sexos, sendo assim, foi adotada uma nova lógica onde se leva em conta a temperatura da água e a sua turbidez. Porém a implementação do sensor de turbidez não foi possível, deixando a escolha da descarga apenas ao sensor de temperatura.

\section{CONCLUSÃO}

Este trabalho aplicou a automatização de um ambiente sanitário simulado por meio de um protótipo desenvolvido a partir de um módulo ESP32, sensores LDR e infravermelho para detectar presença, motor de passo para simular a abertura e o fechamento de um assento de vaso sanitário e uma válvula solenoide para realizar a abertura e fechamento da torneira.

As principais funções da torneira foram aplicadas, sendo possível acionar a torneira com o sensor infravermelho. Em relação ao vaso sanitário, foi desenvolvido um kit para simular a lógica de controle para a abertura e fechamento da tampa do vaso sanitário.

Por fim, espera-se que este projeto contribua de maneira positiva para a crise da pandemia da Covid-19, evitando que novas pessoas sejam contaminadas e, talvez assim, salvando vidas, além de demonstrar que a tecnologia pode ser aplicada à saúde pública, podendo auxiliar a sociedade mesmo em momentos de crise.

Futuramente, pode-se desenvolver o desenvolvimento completo desse projeto e a integração de novas tecnologias, como Internet das Coisas.

\section{REFERÊNCIAS}

AGHENTA, Lawrence Oriaghe; IQBAL, Mohammad Tariq. Low-Cost, Open Source IoTBased SCADA System Design Using Thinger.IO and ESP32 Thing. MDPI Electronics, Basel, 2019.

ARDUINO E CIA. Como Usar o Driver A4988 com Motor de Passo Nema 17. In: Arduino e Cia. [S. l.], 2015. Disponível em: https://www.arduinoecia.com.br/driver-a4988-com-motorde-passo-nema-17/. Acesso em: 2 dez. 2020.

ARMITAGE, Hanae. 'Smart toilet' monitors for signs of disease. In: Stanford Medicine News Center. Stanford, 2020. Disponível em: https://med.stanford.edu/news/allnews/2020/04/smart-toilet-monitors-for-signs-of-disease.html. Acesso em: 8 nov. 2020.

CENTER FOR SYSTEMS SCIENCE AND ENGINEERING AT JOHNS HOPKINS UNIVERSITY. COVID-19 Dashboard. In: Johns Hopkins Coronavirus Research Center. Baltimore, 2021. Disponível em: https://coronavirus.jhu.edu/map.html. Acesso em: 07 jun. 2021. 


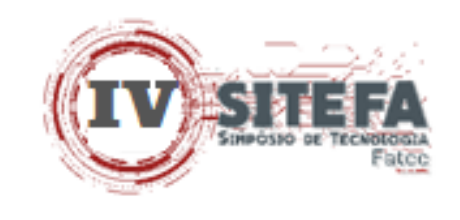

GIL, Antonio Carlos. Como Classificas as Pesquisas com Base em Seus Objetivos? In: Como Elaborar Projetos de Pesquisa. 4. ed. São Paulo: Atlas, 2002. cap. 4, p. 41-57.

LINS, Vitor; MOURA, Waldson. Domótica: Automação Residencial. UNIBRATEC, Recife, 2009.

MAIER, Alexander; SHARP, Andrew; VAGAPOV, Yuriv. Comparative Analysis and Practical. Glyndwr University, Wrexham, 2017.

MARCONI, Marina de Andrade; LAKATOS, Eva Maria. Métodos científicos. In:

Fundamentos da Metodologia Científica. 5. ed. São Paulo: Atlas, 2003. cap. 4, p. 99-100.

RAI, Petrab; REHMAN, Murk. ESP32 Based Smart Surveillance System. In: 2019

International Conference on Computing, Mathematics and Engineering Technologies, 2., 2019, Sukkur. Anais. IEEE, [S. l.: s. n.], 2019. P. 1-3.

RODRIGUES JUNIOR, José Fernando. Pesquisa Experimental. 2021. Apresentação visual digital. Disponível em: http://www.escritacientifica.sc.usp.br/wp-content/uploads/ MPCC_5_DataAnalysis06-PesquisaExperimental.pdf. Acesso em: 01 jul. 2021.

ROSÁRIO, João Maurício. Introdução à automação. In: Automação Industrial. São Paulo: Baraúna, 2009. cap. 1, p. 15-32.

SERKAL, Mariam M. Al. Have you spotted the new smart toilets in Dubai? In: Gulf News. [S. l.], 2019. Disponível em: https://gulfnews.com/uae/have-you-spotted-the-new-smarttoilets-in-dubai-1.1565259563879. Acesso em: 8 nov. 2020.

SILVA, Alexandre Teixeira et al. Ovos de Enterobius vermicularis em Salas de Espera e Banheiros de Unidades Básicas de Saúde (UBS) do Município de Nova Serrana-MG:

Contribuições Para o Controle. Patologia Tropical, [S. l.], 2013. 